\title{
Prolonged survival of patients with EGFR- mutated non-small cell lung cancer with solitary brain metastases treated with surgical resection of brain and lung lesions followed by EGFR TKls
}

\author{
Qi Gui ${ }^{1 \dagger}$, Jiangang Liư ${ }^{2 \dagger}$, Dapeng $\mathrm{Li}^{1}$ and Chengcheng $\mathrm{Xu}^{3^{*}}$
}

\begin{abstract}
Background: The standard combination of initial and subsequent treatments of epidermal growth factor receptor (EGFR)-mutated non-small cell lung cancer (NSCLC) patients with solitary brain metastases (BM) remain unclear. Thus, the management options and the progression-free survival (PFS) and the overall survival (OS) of EGFR-mutated NSCLC patients with solitary BM were investigated in the study.

Methods: We retrospectively reviewed the clinical data from NSCLC patients who harbored EGFR mutations and who presented solitary BM at diagnosis in our institute between 2012 and 2014. PFS and OS were evaluated using Kaplan-Meier methods and compared using log-rank tests.

Results: In total, 36 NSCLC patients with solitary BM who harbored EGFR mutations were enrolled in this study. The PFS and OS of these patients was 12.4 and 19.3 months, respectively. Sixteen patients underwent surgical resection of brain and lung lesions followed by EGFR-TKIs treatment, and the median OS was 28. 0 months, which was significantly longer than 16.4 months of 14 patients received radiotherapy combined with or followed by EGFR-tyrosine kinase inhibitors (TKIs) and 15.8 months of 6 patients received radiotherapy followed by chemotherapy. The median PFS also showed the same trend in each group (16.1, 10.4, and 9. 8 months, respectively).

Conclusions: The survival was extended in the patients receiving surgical resection of brain and lung lesions followed by EGFR-TKIs treatment, and surgery combined with EGFR-TKls could be a recommended treatment for EGFR mutated NSCLC patients with solitary BM.
\end{abstract}

Keywords: Brain metastases, Non-small cell lung cancer, Epidermal growth factor receptor, Surgical resection

\section{Background}

Brain metastases (BM) continue to be one of the most crucial distant metastases in patients with NSCLC, which cause deterioration in quality of life (QOL) and limited life expectancy. Approximately 10-20\% NSCLC patients presented $\mathrm{BM}$ at diagnosis [1]. Furthermore, if

\footnotetext{
* Correspondence: xuchengcheng@suda.edu.cn

${ }^{\dagger}$ Equal contributors

${ }^{3}$ Departments of Thoracic Surgery, The First Affiliated Hospital of Soochow University, Suzhou 215006, Jiangsu, People's Republic of China

Full list of author information is available at the end of the article
}

all NSCLC patients had undergone MRI brain at the time of diagnosis, $3 \%$ of stage I/II patients and $21 \%$ of stage IIIA patients could have been found BM [2].

When NSCLC patients developed BM, the prognosis remains poor with the OS less than 3 months without any treatment [3]. For metastatic disease, systemic therapy is the standard management. However, for NSCLC patients with solitary BM, local treatments of brain and lung lesions may represent useful approaches with a slight improvement in terms of survival, local control, and symptom relief. Three most used treatment options 
are available for management of brain lesion, including whole brain radiation therapy (WBRT), surgery, and/or stereotactic radiosurgery (SRS). A general consensus has not yet been reached as to which treatment is most effective in such patients. Moreover, up to $40-50 \%$ of lung adenocarcinoma patients of East Asian descent harbor EGFR mutations [4]. Previous studies suggested that NSCLC patients with EGFR mutation had higher incidence of BM $[5,6]$. Based on the time of discovery of EGFR mutation, these patients may receive EGFR TKIs or chemotherapy as subsequent therapy. There are currently no reported data on which is best comprehensive management approach and PFS as well as OS of EGFRmutated NSCLC patients with solitary BM. Therefore, in the present study, PFS and OS of EGFR-mutated NSCLC patients with solitary BM treated with surgery or radiation therapy followed by EGFR-TKIs or chemotherapy were investigated.

\section{Methods \\ Patients}

Medical data were retrospectively reviewed from NSCLC patients who harbored EGFR mutations and who presented solitary BM at diagnosis in our institute between January 2012 and December 2014. A total of 72 NSCLC patients with solitary BM were evaluated EGFR mutation status in lung biopsy specimens or malignant pleural effusion or bronchoalveolar lavage fluid in our hospital center laboratory. The screening was carried out by polymerase chain reaction (PCR) clamp method. Of these 72 patients, 33 were ineligible because of EGFR wild type and 1 was excluded because of the exon 20T790M mutation, which correlated with resistance to EGFR-TKIs. Twenty patients were detected exon 19 deletion, 17 patients presented exon 21 L858R point mutations, and 1 patient was determined exon 18 G719X point mutation.

Enrolled patients were followed up every 2-3 months and were evaluated based on Response Evaluation Criteria in Solid Tumors (RECIST 1.1). Two of 38 enrolled patients dropped out. Eventually, the information of 36 patients were extracted regarding gender, age, ECOG performance status (PS), recursive partitioning analysis (RPA), graded prognostic assessment (GPA), EGFR mutation types, EGFR-TKI, radiotherapy strategies, date of disease progression and last follow-up, and the outcome of patients from the records.

\section{Statistical analysis}

OS was defined as the time from diagnosis until death or last follow-up. And PFS was defined as the time from the date of initiation of any treatment of surgery or radiotherapy or EGFR-TKIs to the date of documented progression or death. The last follow-up was on 31 December 2016. Categorical variables were compared using Fisher's exact test. OS and PFS were compared using Log-rank analysis and assessed using the Kaplan-Meier method. The $p$ value less than 0.05 was considered statistically significant. Analyses were performed using SPSS 16 software.

\section{Results}

\section{Patients' characteristics}

Thirty-six patients with solitary BM who harbored EGFR mutation were included (Fig. 1), and their characteristics are summarized in Table 1. There were $12(33.3 \%)$ males and $24(66.7 \%)$ females with a median age of $58(39-72)$ years. The most patients were ECOG PS 0-1 (88.9\%). Twenty-seven (75\%) patients were assessed at RPA I, and the major GPA score at diagnosis was 2.5-3 (20 patients, 55.6\%). Among these 36 patients, 15 were with exon 21 L858R point mutations, 20 with deletion in exon 19, and 1 with other mutation (exon 18 G719X mutation). Twentyone patients received gefitinib as their EGFR-TKI, while the others ( 9 cases) received erlotinib. For radiotherapy of brain mass, 16 patients chose SRS and 4 patients chose WBRT. After diagnosis, 16 patients (SUR + TKI group) underwent consecutive surgical resection of brain and lung lesions followed by EGFR-TKIs treatment, among whom 1 patient presented EGFR wild type of brain specimen. Among these patients, 12 patients were diagnosed $c \& \mathrm{pT}_{1-3} \mathrm{~N}_{0-1} \mathrm{M}_{1 \mathrm{~b}}$, and 4 patients were diagnosed $\mathrm{cT}_{1-3} \mathrm{~N}_{0-1} \mathrm{M}_{1 \mathrm{~b}}$ before surgery and ${ }_{\mathrm{P}} \mathrm{T}_{1-3} \mathrm{~N}_{2} \mathrm{M}_{1 \mathrm{~b}}$ from pathologic information after surgery. And 14 patients (RT + TKI group) received radiotherapy combined with or followed by EGFR-TKIs. Moreover, 6 patients (RT + CHE group) received radiotherapy followed by chemotherapy. Among these patients, 2 patients received pemetrexed + carboplatin, 4 patients received pemetrexed + cisplatin. For all aspects of baseline characteristics, there were no statistically significant differences among each group above $(p>0.05)$.

\section{Survival analysis}

The median PFS in all 36 patients was 12.4 months (range 5.8-32.1), while the PFS of the patients in SUR + TKI group, RT + TKI group and RT + CHE group were 16.1 months (95\%CI 10.1-21.9), 10.4 months (95\% CI 3.816.2 ), and 9.8 months (95\% CI 7.8-12.1), respectively (Fig. 2a). And the PFS of SUR + TKI group was significantly longer than that of RT + CHE group $(p=0.044)$. No PFS difference was found between SUR + TKI patients and RT + TKI patients $(p=0.136)$, and between RT + TKI patients and RT + CHE patients $(p=0.685)$.

The median survival time of all the patients was 19.3 months (range 11.2-40.3). The OS in SUR + TKI group, RT + TKI group and RT + CHE group were 28.0 months (95\% CI 19.2-36.8), 16.4 months (95\% CI 10.7-21.3), and 15.8 months (95\% CI 15.1-16.9), respectively (Fig. 2b). The patients in SUR + TKI group had prolonged survival than that in RT + TKI group 


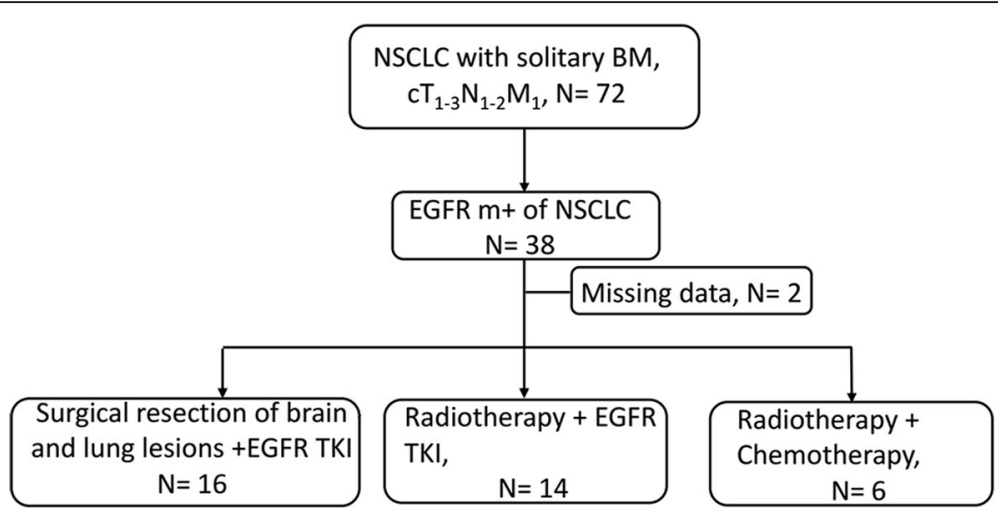

Fig. 1 Patient profile

$(p=0.048)$ and than that in $\mathrm{RT}+\mathrm{CHE}$ group $(p=0.025)$. No OS difference was found between $\mathrm{RT}+\mathrm{TKI}$ patients and RT + CHE patients $(p=0.842)$.

\section{Discussion}

BM of NSCLC is a frequent complication of NSCLC, which remains a major challenge with relatively limited survival benefits with current therapy strategies. In general, radiotherapy of $\mathrm{BM}$ in combination with systemic therapy (chemotherapy and/or molecular target therapy) is the most widely used approach for NSCLC patients with BM. Previous study demonstrated that systemic chemotherapy for NSCLC patients with BM had an OS of 68 months [7]. And the median OS of NSCLC patients with BM harboring EGFR mutations treated with EGFR-TKIs is in the range of 15-20 months, which significantly longer than that of EGFR wild-type tumors [8, 9]. In this present study, the median PFS and OS of EGFR mutated
NSCLC patients with solitary BM treated with EGFR-TKIs (SUR + TKI group and RT + TKI group) was 13.1 and 20.4 months, respectively, which is similar to previous researches. To our knowledge, this is the first study of compared management options and calculated the PFS and OS of EGFR mutated NSCLC patients with solitary BM.

Notably, for patients with solitary BM, the OS may be different from that with multiple metastases, and the local treatment may be the first option. A study showed that 74.6\% NSCLC patients with solitary BM survived more than 1 year, and single brain metastasis is commonly considered to be a favorable prognostic factor [10]. It was well accepted that the standard treatment of NSCLC patients with solitary BM was surgical resection of solitary brain lesions and with WBRT and/or SRS. Tissue diagnosis is still the gold standard as pathological diagnosis. As early as 1990, a study showed that 6 of 59 patients thought to BM actually had an abscess, benign tumor or another primary

Table 1 Patient characteristics

\begin{tabular}{|c|c|c|c|c|c|}
\hline & Total (N) & SUR + TKI (N) & RT + TKI (N) & $\mathrm{RT}+\mathrm{CHE}(\mathrm{N})$ & $p$ \\
\hline Male/female & $12 / 24$ & $6 / 10$ & $4 / 10$ & $2 / 4$ & 0.884 \\
\hline \multicolumn{6}{|l|}{ Age } \\
\hline Median (range) & 58 (39-72) & 56.5 (39-68) & $57.5(45-72)$ & $59(46-63)$ & 0.767 \\
\hline \multicolumn{6}{|l|}{ ECOG PS } \\
\hline $0 / 1 / 2 / 3 / 4$ & 20/12/4/0 & $12 / 4 / 0 / 0$ & $6 / 5 / 3 / 0$ & $2 / 3 / 1 / 0$ & 0.057 \\
\hline \multicolumn{6}{|l|}{ RPA } \\
\hline$|/||/|||$ & $27 / 5$ & $14 / 2 / 0$ & $8 / 3 / 3$ & $5 / 0 / 1$ & 0.114 \\
\hline \multicolumn{6}{|l|}{ GPA } \\
\hline $1-2 / 2.5-3 / 3.5-4$ & $2 / 20 / 14$ & $0 / 7 / 9$ & $2 / 8 / 4$ & $0 / 5 / 1$ & 0.264 \\
\hline EGFR m + type & & 6/10/0 (lung) & & & \\
\hline 21/19/others & 15/20/1 & 6/9/0 (brain) & $7 / 6 / 1$ & $2 / 4 / 0$ & 0.934 \\
\hline \multicolumn{6}{|l|}{ EGFR-TKI } \\
\hline Gefitinib/erlotinib & $21 / 19$ & $12 / 4$ & $9 / 5$ & - & 0.539 \\
\hline \multicolumn{6}{|l|}{ RT } \\
\hline SRS/WBRT & $16 / 4$ & - & $12 / 2$ & $4 / 2$ & 0.355 \\
\hline
\end{tabular}




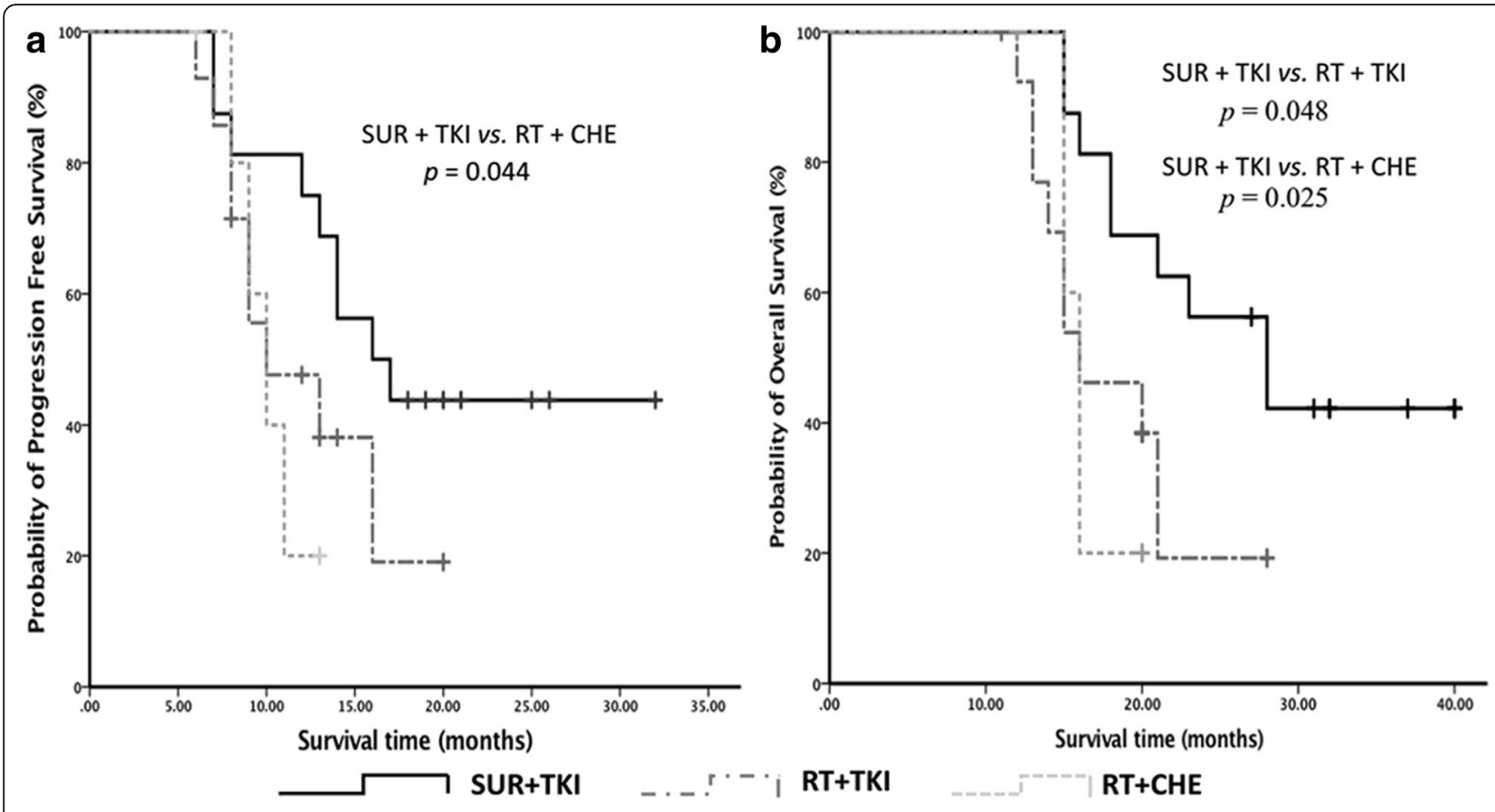

Fig. 2 Kaplan-Meier Plot. a progression-free survival (PFS). b Overall survival (OS)

tumor [11]. This case indicates that some brain lesions thought to be metastases of NSCLC might be misdiagnosed and received invalid treatments. Furthermore, a case report of a patient whose primary lesion harbored an exon 20T790M mutation, while the BM specimen was negative for the mutation [12]. And in this present study, 1 patient presented deletion in exon 19 of lung lesion and EGFR wild type of brain lesion. These all indicates that the gene performance of BM may differ from that of the primary lesion, and surgery of BM will help not only to remove of cranial lesion but also to allow exact tissue and gene diagnosis and indicate the best management approach. Additionally, in this study, patients receiving surgical resection of brain and lung lesions followed by EGFR-TKIs treatment gained significantly longer survival than radiotherapy combined with EGFR-TKIs or radiotherapy in combination with chemotherapy. These data suggest that surgical intervention combined with EGFR-TKIs is beneficial for EGFR mutated NSCLC patients with solitary BM.

Another explanation for the survival benefit of surgical intervention combined with EGFR-TKIs group is that patients in this group underwent surgeries of lung tumor. Several series have previously reported encouraging survival outcomes after lung surgery in patients with synchronous BM [13, 14], and our findings supported this conclusion. Moreover, EGFR-TKIs treatment is another possible beneficial factor, which is the standard management as first line of advanced NSCLC patients harboring EGFR mutations. Recently, an increasing interest has been presented in the role of EGFR-TKIs in the treatment of
BM from NSCLC. Evidences suggest that EGFR-TKIs can cross the blood-brain barrier (BBB) [15], while most chemotherapeutic agents cannot cross BBB. However, for the patients who underwent surgery of both brain and lung lesions, whether EGFR-TKIs are suitable as adjuvant treatment remains unclear, while currently there was no strong evidence of EGFR-TKIs as adjuvant treatment of local NSCLC. In this study, patients with surgery of both brain and lung lesions followed by EGFR-TKIs had prolonged survival, which supported that EGFR-TKIs are recommended as adjuvant treatment of EGFR mutated NSCLC patients with solitary BM.

In this study, WBRT was not used as initial therapy followed surgery of BM. When 10 patients presented recurrence of brain disease several months later, they received WBRT at that time. A retrospective study demonstrated that WBRT of patients had deleterious results [16]. Moreover, another study showed that compared to the WBRT group, patients in the observation group had better mean scores in physical, role, and cognitive functioning at 8 weeks, and thus the WBRT can be delayed [17].

The most remarkable limitation of this study is that the study conducted at a single institution and the number of patients is relatively small. Secondly, subsequent therapy and/or further therapy could not be fully evaluated due to limited patients sample size. However, as the first study investigating the role of management options and the PFS and OS of EGFR mutated NSCLC patients with solitary BM, the findings are clinically meaningful. 


\section{Conclusions}

The survival was prolonged in the patients receiving surgical resection of brain and lung lesions followed by EGFR-TKIs treatment, in comparison with that received radiotherapy of BM combined with EGFR-TKIs or radiotherapy of BM followed by chemotherapy. Surgery combined with EGFRTKIs could be a recommended treatment for EGFR mutated NSCLC patients with solitary BM. In the future, larger and prospective studies will be needed to confirm these results.

\begin{abstract}
Abbreviations
BM: brain metastases; ECOG: Eastern Cooperative Oncology Group; EGFR: Epidermal growth factor receptor; GPA: Graded prognostic assessment; m + : Mutation-positive; NSCLC: Non-small cell lung cancer; OS: Overall survival; PCR: Polymerase chain reaction; PFS: Progression free survival; PS: Performance status; QOL: Quality of life; RPA: Recursive partitioning analysis; RT: Radiotherapy; SRS: Stereotactic radiosurgery; SUR: Surgical resection of brain and lung lesions; TKIs: Tyrosine kinase inhibitors; WBRT: Whole brain radiation therapy
\end{abstract}

\section{Acknowledgements}

Not applicable.

\section{Funding}

This study was supported by the National Natural Science Foundation of China (Grant no.81502275)

\section{Availability of data and materials}

All data are fully available without restriction.

\section{Authors' contributions}

CX designed the study and modified the paper. QG performed the result analysis and wrote the paper. $J L$ collected the patients' data and made the follow-up. $J \mathrm{~L}$ and $\mathrm{DL}$ performed the data analysis. All authors read and approved the final manuscript.

\section{Ethics approval and consent to participate}

This study was approved by Ethics Committee of First Affiliated Hospital of Soochow University. Patient data were de-identified and anonymized before analysis.

\section{Consent for publication}

Informed consent for publication was obtained in all cases (patients or their relative, if the patients have died). They all understand that this journal is freely available on the web and under the license which the journal uses material published can be redistributed freely and used for any legal purpose.

\section{Competing interests}

The authors declare that they have no competing interests.

\section{Publisher's Note}

Springer Nature remains neutral with regard to jurisdictional claims in published maps and institutional affiliations.

\begin{abstract}
Author details
${ }^{1}$ Departments of Oncology, The First Affiliated Hospital of Soochow University, Suzhou 215006, Jiangsu, People's Republic of China. ${ }^{2}$ Departments of Neurosurgery, The First Affiliated Hospital of Soochow University, Suzhou 215006, Jiangsu, People's Republic of China. ${ }^{3}$ Departments of Thoracic Surgery, The First Affiliated Hospital of Soochow University, Suzhou 215006, Jiangsu, People's Republic of China.
\end{abstract}

Received: 19 April 2017 Accepted: 28 August 2017

Published online: 16 October 2017

\section{References}

1. Mujoomdar A, Austin JH, Malhotra R, et al. Clinical predictors of metastatic disease to the brain from non-small cell lung carcinoma: primary tumor size, cell type, and lymph node metastases. Radiology. 2007;242:882-8.
2. Hochstenbag MM, Twijnstra A, Hofman P, Wouters EF, ten Velde GP. MRimaging of the brain of neurologic asymptomatic patients with large cell or adenocarcinoma of the lung. Does it influence prognosis and treatment? Lung Cancer. 2003;42:189-93.

3. Nussbaum ES, Djalilian HR, Cho KH, Hall WA. Brain metastases. Histology, multiplicity, surgery, and survival. Cancer. 1996;78:1781-8.

4. Shi Y, Au JS, Thongprasert S, Srinivasan S, Tsai CM, Khoa MT, Heeroma K, Itoh Y, Cornelio G, Yang PC. A prospective, molecular epidemiology study of EGFR mutations in Asian patients with advanced non-small-cell lung cancer of adenocarcinoma histology (PIONEER). J Thorac Oncol. 2014;9:154-62.

5. Han G, Bi J, Tan W, Wei X, Wang X, Ying X, Guo X, Zhou X, Hu D, Zhen W. A retrospective analysis in patients with EGFR-mutant lung adenocarcinoma: is EGFR mutation associated with a higher incidence of brain metastasis? Oncotarget. 2016;35:56998-7010.

6. Stanic K, Zwitter M, Hitij NT, Kern I, Sadikov A, Cufer T. Brain metastases in lung adenocarcinoma: impact of EGFR mutation status on incidence and survival. Radiol Oncol. 2014;48:173-83.

7. Moscetti L, Nelli F, Felici A, Rinaldi M, De Santis S, D'Auria G, et al. Up-front chemotherapy and radiation treatment in newly diagnosed treatment nonsmall cell lung cancer with brain metastases: survey by outcome research network for evaluation of treatment results in oncology. Cancer. 2007:109:274-81.

8. Park SJ, Kim HT, Lee DH, Kim KP, Kim SW, Suh C, Le JS. Efficacy of epidermal growth factor receptor tyrosine kinase inhibitors for brain metastases in non-small-cell lung cancer harbouring either exon 19 or 21 mutation. Lung Cancer. 2012;77:556-60.

9. Welsh JW, Komaki R, Amini A, Munsell MF, Unger W, Allen PK, Chang JY, et al. Phase II trial of erlotinib plus concurrent whole-brain radiation therapy for patients with brain metastases from non-small-cell lung cancer. J Clin Oncol. 2013;31:895-902.

10. Connolly EP, Mathew M, Tam M, King JV, Kunnakkat SD, Parker EC, Golfinos JG, Gruber ML, Narayana A. Involved field radiation therapy after surgical resection of solitary brain metastases - mature results. Neuro-Oncology. 2013;15:589-94.

11. Patchell RA, Tibbs PA, Walsh JW, Dempsey RJ, Maruyama Y, Kryscio RJ, Markesbery WR, Macdonald JS, Young B. A randomized trial of surgery in the treatment of single metastases to the brain. N Engl J Med. 1990;322:494-500.

12. Ohara S, Ushijima T, Gunji M, Tanai C, Tanaka Y, Noda H, et al. Brain metastasis effectively treated with erlotinib following the acquisition of resistance to gefitinib: a case report. J Med Case Rep. 2014;8:1-5.

13. Bonnette P, Puyo P, Gabriel C, et al. Surgical management of non-small cell lung cancer with synchronous brain metastases. Chest. 2001;119:1469-75.

14. Lind JS, Lagerwaard FJ, Smit EF, et al. Time for reappraisal of extracranial treatment options? Synchronous brain metastases from nonsmall cell lung cancer. Cancer. 2011;117:597-605.

15. Zhao J, Chen M, Zhong W, Zhang L, Li L, Xiao Y, Nie L, Hu P, Wang M. Cerebrospinal fluid concentrations of gefitinib in patients with lung adenocarcinoma. Clin Lung Cancer. 2013;14:188-93.

16. Ebi J, Sato H, Nakajima M, Shishido F. Incidence of leukoencephalopathy after whole-brain radiation therapy for brain metastases. Int J Radiat Oncol Biol Phys. 2013;85:1212-7.

17. Soffietti R, Kocher M, Abacioglu UM, Villa S, Fauchon F, Baumert BG, et al. A European organisation for research and treatment of cancer phase III trial of adjuvant whole-brain radiotherapy versus observation in patients with one to three brain metastases from solid tumours after surgical resection or radio- surgery: quality-of-life results. J Clin Oncol. 2013;31:65-72.

Submit your next manuscript to BioMed Central and we will help you at every step:

- We accept pre-submission inquiries

- Our selector tool helps you to find the most relevant journal

- We provide round the clock customer support

- Convenient online submission

- Thorough peer review

- Inclusion in PubMed and all major indexing services

- Maximum visibility for your research

Submit your manuscript at www.biomedcentral.com/submit
Ciomed Central 